\title{
AUTOMORPHISMS OF GRASSMANNIANS
}

\author{
MICHAEL J. COWEN
}

(Communicated by Irwin Kra)

\begin{abstract}
For a complex vector space $\mathscr{V}$ of dimension $n$, the group of holomorphic automorphisms of the Grassmannian $\operatorname{Gr}(p, \mathscr{V})$ can be identified with the subgroup of $\mathrm{PGl}\left(\bigwedge^{p} \mathscr{V}\right)$ preserving the Grassmannian. Using this, Chow showed $\operatorname{Aut}(\operatorname{Gr}(p, \mathscr{V}))=\mathbf{P G l}(\mathscr{V})$ for $n \neq 2 p$, and $\mathbf{P G l}(\mathscr{V})$ is a normal subgroup of index 2 in $\operatorname{Aut}(\operatorname{Gr}(p, \mathscr{V}))$ for $n=2 p$. We prove a version of Chow's result for a separable Hilbert space $\mathscr{H}$. Theorem. $\mathbf{P G l}(\mathscr{H})$ is the subgroup of $\operatorname{PGl}\left(\bigwedge^{p} \mathscr{H}\right)$ which preserves $\operatorname{Gr}(p, \mathscr{H})$. That is, if $R$ is an invertible linear operator on $\bigwedge^{p} \mathscr{H}$ which preserves decomposable $p$-vectors, then there exists $S$, an invertible linear operator on $\mathscr{H}$, such that $R=\bigwedge^{p} S$.
\end{abstract}

\section{INTRODUCTION}

Let $\mathscr{V}$ be a complex vector space of dimension $n$. Then a holomorphic automorphism of $\operatorname{Gr}(p, \mathscr{V})$, the Grassmannian of $p$-planes in $\mathscr{V}$, is induced by an endomorphism of $\Lambda^{p} \mathscr{V}$ preserving decomposable $p$-vectors: $\operatorname{Aut}(\operatorname{Gr}(p, \mathscr{V}))$ $=\operatorname{PGl}\left(\Lambda^{p} \mathscr{V}\right)_{\operatorname{Gr}(p, \mathscr{V})}$, the subgroup of $\operatorname{PGl}\left(\Lambda^{p} \mathscr{V}\right)$ preserving the Grassmannian. For example, $S$ in $\mathrm{Gl}(\mathscr{V})$ induces an automorphism $\Phi_{S}$ of $\operatorname{Gr}(p, \mathscr{V})$, by sending a $p$-plane $W$ into $S W$. Classically, $\Phi_{S}$ is called a collineation; the corresponding endomorphism of $\Lambda^{p} \mathscr{V}$ is $\Lambda^{p} S$. Chow [C] showed all automorphisms are collineations, except when the dual map $*: \operatorname{Gr}(p, \mathscr{V}) \rightarrow \operatorname{Gr}(n-p, \mathscr{V})$ is an automorphism-when $n=2 p$. In that case, there are the additional automorphisms of the form $* \circ \Phi_{S}$, the correlations:

Theorem 1.1 (Chow). $\operatorname{Aut}(\operatorname{Gr}(p, \mathscr{V}))=\operatorname{PGl}(\mathscr{V})$ for $\operatorname{dim} \mathscr{V} \neq 2 p . \operatorname{PGl}(\mathscr{V})$ is a normal subgroup of index 2 in $\operatorname{Aut}(\operatorname{Gr}(p, \mathscr{V}))$ for $\operatorname{dim} \mathscr{V}=2 p$.

Using the Schubert calculus, Tango [T] gave an alternative proof to Theorem 1.1, generalizing Chow's result to automorphisms of flag manifolds. Kaup [K] used Lie theory to study the Grassmannian of a Banach space. In particular, he showed for a Hilbert space $\mathscr{H}$, every automorphism of $\operatorname{Gr}(p, \mathscr{H})$ is a collineation when $1 \leq p<\infty$.

Received by the editors December 1, 1987.

1980 Mathematics Subject Classification (1985 Revision). Primary 32M05; Secondary 14M15.

This research was supported in part by NSF Grant DMS-8602197. 
For $\mathscr{H}$ a separable Hilbert space, we consider the holomorphic map

$$
\varphi_{R}: \operatorname{Gr}(p, \mathscr{H}) \rightarrow \operatorname{Gr}(p, \mathscr{H}),
$$

induced by an invertible linear operator $R$ on $\bigwedge^{p} \mathscr{H}$ which preserves decomposable $p$-vectors. Such operators arise naturally when studying holomorphic curves in $\operatorname{Gr}(p, \mathscr{H})$ which have the same curvature invariants. We denote by $\operatorname{PGl}\left(\bigwedge^{p} \mathscr{H}\right)_{\mathrm{Gr}(p, \mathscr{H})}$ the subset of $\operatorname{PGl}\left(\bigwedge^{p} \mathscr{H}\right)$ consisting of all $\varphi_{R}$ which preserve the Grassmannian. If $\mathscr{H}$ is finite dimensional, then $\operatorname{Gr}(p, \mathscr{H})$ is compact and connected, so $\varphi_{R}$ is an automorphism. When $\mathscr{H}$ is infinite dimensional, it does not follow directly from our assumptions that $R^{-1}$ preserves the Grassmannian (so that $\operatorname{PGl}\left(\bigwedge^{p} \mathscr{H}\right)_{\operatorname{Gr}(p, \mathscr{H})}$ is a subgroup of $\operatorname{Aut} \operatorname{Gr}(p, \mathscr{H})$.) Nonetheless, we prove a version of Chow's Theorem which shows

$$
\operatorname{PGl}\left(\bigwedge^{p} \mathscr{H}\right)_{\operatorname{Gr}(p, \mathscr{H})}
$$

is indeed a subgroup of $\operatorname{Aut} \operatorname{Gr}(p, \mathscr{H})$ :

Theorem 1.2. $\mathrm{PGl}\left(\bigwedge^{p} \mathscr{H}\right)_{\mathrm{Gr}(p, \mathscr{H})}=\mathrm{PGl}(\mathscr{H})$. That is, if $R$ is an invertible linear operator on $\Lambda^{p}(\mathscr{H})$. which preserves decomposable p-vectors, then $R$ is a collineation: $R=\Lambda^{p} S$ for an invertible linear operator $S$ on $\mathscr{H}$.

Note that $R$ determines $S$ up to multiplication by a scalar: $S x$ spans $\bigcap_{x \in W} \varphi_{R}(W)$, while $S x$ and $\widetilde{S} x$ dependent for all $x$ implies $\widetilde{S}=c S$. Consequently, if $R$ is unitary, then $\bigwedge^{p}\left(S^{*} S\right)=I$ implies $S^{*} S=c I$, so we have the following corollary:

Corollary 1.3. If $\widetilde{U}$ is unitary on $\wedge^{p} \mathscr{H}$ and preserves decomposable p-vectors, then $\widetilde{U}=\Lambda^{p} U$ for $U$ unitary on $\mathscr{H}$.

The proof of Theorem 1.2 uses only elementary linear algebra. Along the way we give a short proof of Chow's result using one additional well-known but non-elementary fact: the Picard group of the Grassmannian is $\mathbf{Z}$.

\section{Preliminaries}

That Aut $\operatorname{Gr}(p, \mathscr{V})$ equals $\operatorname{PGl}\left(\bigwedge^{p} \mathscr{V}\right)_{\operatorname{Gr}(p, \mathscr{V})}$ for $\mathscr{V}$ finite dimensional can be proved along the lines of the $\mathbf{P}_{n}$ case as found in [H]. The crucial fact needed is that the group of equivalence classes of holomorphic line bundles on $\operatorname{Gr}(p, \mathscr{V})$ - the Picard group-is isomorphic to $\mathbf{Z}$. This follows from the homology of the Grassmannian in terms of Schubert cycles and from the Hodge decomposition: $H^{1}(\operatorname{Gr}(p, \mathscr{V}), \mathscr{A})$ equals $H^{2}(\mathrm{Gr}(p, \mathscr{V}), \mathscr{A})=0$, where $\mathscr{A}$ is the sheaf of germs of analytic functions; thus $H^{1}\left(\operatorname{Gr}(p, \mathscr{V}), \mathscr{A}^{*}\right)$ $\cong H^{2}(\operatorname{Gr}(p, \mathscr{V}), \mathbf{Z}) \cong \mathbf{Z}$.

To fix a choice of the dual map $*$ : let $e_{1}, \ldots, e_{n}$ be a basis for $\mathscr{V}$ and $\delta_{1}, \ldots, \delta_{n}$ the dual basis. Define $l: \mathscr{V}^{*} \rightarrow \mathscr{V}$ by $l\left(\delta_{j}\right)=e_{j}$, so that $j \circ \imath=l^{t}$, where $j: \mathscr{V} \rightarrow \mathscr{V}^{* *}$ is the identification map. Then $l$ induces a biholomorphic mapping, the dual map $*: \operatorname{Gr}(p, \mathscr{V}) \rightarrow \operatorname{Gr}(n-p, \mathscr{V})$, as follows: if $W$ is a 
$p$-plane, then $* W=\imath\left(W^{\perp}\right)$, where $W^{\perp}$ is the annihilator of $W$. Note $*$ is induced by an isomorphism of $\Lambda^{p} \mathscr{V} \rightarrow \Lambda^{n-p} \mathscr{V}$ (a basis $p$-vector $e_{I}$ maps to the $(n-p)$-vector $\varepsilon_{I J} e_{J}$, where $\left.e_{I} \wedge e_{J}=\varepsilon_{I J} e_{1} \wedge \cdots \wedge e_{n}\right)$. Furthermore, if $S: \mathscr{V}^{*} \rightarrow \mathscr{V}$ is a linear transformation, then $S\left(W^{\perp}\right)=\left(\left(S^{t}\right)^{-1} j W\right)^{\perp}$, so the following holds:

Lemma 2.1. Let $T$ be in $\mathrm{Gl}(\mathscr{V})$, and let $\Phi_{T}$ be the induced automorphism on $\operatorname{Gr}(p, \mathscr{V})$. Then $* \circ \Phi_{T} \circ *$ is the automorphism of $\operatorname{Gr}(n-p, \mathscr{V})$ which is induced by $\imath \circ\left(T^{t}\right) \circ l^{-1} \in \mathrm{Gl}(\mathscr{V})$. In particular, $*^{2}=\mathrm{Id}$.

Remark 2.2. If $\varphi \in \operatorname{Aut}(\operatorname{Gr}(p, \mathscr{V}))$, then $* \circ \varphi \circ * \in \operatorname{Aut}(\operatorname{Gr}(n-p, \mathscr{V}))$. It thus suffices to prove Theorem 1.1 for $\operatorname{dim} \mathscr{V} \geq 2 p$.

\section{ADJACENCY}

Let $R: \bigwedge^{p} \mathscr{H} \rightarrow \Lambda^{p} \mathscr{H}$ be a (bounded) invertible linear operator which preserves the decomposable elements of $\Lambda^{p} \mathscr{H}$ (those of the form $v_{1} \wedge \cdots \wedge v_{p}$ ), and let $\varphi_{R}: \operatorname{Gr}(p, \mathscr{H}) \rightarrow \operatorname{Gr}(p, \mathscr{H})$ be the induced map. The most important tool for analyzing $R$ is what Chow calls adjacency: distinct $p$-planes $V$ and $W$ are adjacent if $\operatorname{dim} V \cap W=p-1$.

Lemma 3.1. Let $V$ and $W$ be p-planes in $\mathscr{H}$ with $\alpha$ and $\beta$ the corresponding decomposable p-vectors in $\Lambda^{p} \mathscr{H}$. Then $V$ and $W$ are adjacent if and only if $\alpha+\beta$ is decomposable.

Proof. Assume that $\alpha+\beta$ is decomposable-the implication in the other direction is trivial. If $\operatorname{dim} V \cap W=q<p-1, \bmod$ out by $V \cap W$ and replace $\alpha$ and $\beta$ by appropriate $(p-q)$-vectors; the problem reduces to showing $\operatorname{dim} V \cap W$ cannot equal 0 if $p>1$ and $\alpha+\beta$ is decomposable.

Assume that $\operatorname{dim} V \cap W=0$; then $V+W$ is $2 p$-dimensional. Now $\alpha+\beta$ is in $\Lambda^{p}(V+W)$, which implies that $\alpha+\beta=\left(v_{1}+w_{1}\right) \wedge \cdots \wedge\left(v_{p}+w_{p}\right)$, where $v_{i} \in V$ and $w_{i} \in W$. Since $\alpha \wedge\left(w_{1} \wedge \cdots \wedge w_{p}\right)=\alpha \wedge(\alpha+\beta)=\alpha \wedge \beta$, then $\beta=w_{1} \wedge$ $\cdots \wedge w_{p}$, and similarly $\alpha=v_{1} \wedge \cdots \wedge v_{p}$; in particular, $v_{1}, \ldots, v_{p}, w_{1}, \ldots, w_{p}$ are independent. But $\alpha+\beta=v_{1} \wedge \cdots \wedge v_{p}+v_{1} \wedge w_{1} \wedge \cdots \wedge v_{p}+\cdots+w_{1} \wedge \cdots \wedge w_{p}$, which implies the mixed terms $v_{1} \wedge w_{1} \wedge \cdots \wedge v_{p}$, etc. sum to zero, a contradiction to independence of the $v_{i}$ and $w_{i}$. Thus $\operatorname{dim} V \cap W$ cannot equal 0 .

Remark 3.2. If $\varphi_{R}: \operatorname{Gr}(p, \mathscr{H}) \rightarrow \operatorname{Gr}(p, \mathscr{H})$ is induced by $R: \Lambda^{p} \mathscr{H} \rightarrow \Lambda^{p} \mathscr{H}$ linear and preserving decomposables, then $\varphi_{R}$ preserves adjacency.

Proof. If $\operatorname{dim} V \cap W=p-1$, let $\mu \in \Lambda^{p-1} \mathscr{H}$ correspond to $V \cap W$; then $V$ corresponds to $\mu \wedge v$ for $v \in \mathscr{H}$ and $W$ corresponds to $\mu \wedge w$. Thus $R(\mu \wedge(v+w))$ is decomposable and the result follows from Lemma 3.1.

\section{Preserving/Dualizing Schubert cycles}

Let $V$ be a $q$-plane in $\mathscr{H}$. For $p>q$, denote by $\sigma_{p}(V)$ the Schubert cycle defined by

$$
\sigma_{p}(V)=\{W \in \operatorname{Gr}(p, \mathscr{H}) \mid V \subset W\},
$$


and for $p<q$ denote by $\Sigma^{p}(V)$ the Schubert cycle defined by $\Sigma^{p}(V)=$ $\{W \in \operatorname{Gr}(p, \mathscr{H}) \mid W \subset V\}$.

Proposition 4.1. Let $\psi: \operatorname{Gr}(p, \mathscr{H}) \rightarrow \operatorname{Gr}(p, \mathscr{H})$ be a map (not necessarily continuous) preserving adjacency. Let $V$ be $a(p-1)$-plane, $W_{1}$ and $W_{2}$ distinct p-planes containing $V$. Denote by $\psi_{p-1}(V)$ the $(p-1)$-plane $\psi\left(W_{1}\right) \cap$ $\psi\left(W_{2}\right)$ and by $\psi^{p+1}(V)$ the $(p+1)$-plane $\psi\left(W_{1}\right)+\psi\left(W_{2}\right)$. Then either (i) $\psi$ preserves the Schubert cycle: $\psi\left(\sigma_{p}(V)\right) \subset \sigma_{p}\left(\psi_{p-1}(V)\right)$, so that $\psi_{p-1}(V)$ is independent of the choice of $W_{1}$ and $W_{2}$; or (ii) $\psi$ dualizes the Schubert cycle: $\psi\left(\sigma_{p}(V)\right) \subset \Sigma^{p}\left(\psi^{p+1}(V)\right)$, so that $\psi^{p+1}(V)$ is independent of the choice. Proof. If (i) does not hold, then there exists a $p$-plane $W_{3}$ containing $V$ such that $\psi\left(W_{3}\right)$ does not contain $\psi_{p-1}(V)$. Let $\psi\left(W_{i}\right)=\widetilde{W}_{i}$; then $\widetilde{W}_{3}$ intersects $\widetilde{W}_{1}$ and $\widetilde{W}_{2}$ in different $(p-1)$-planes-otherwise $\widetilde{W}_{1} \cap \widetilde{W}_{2} \cap \widetilde{W}_{3}=\psi_{p-1}(V)$ and hence $\widetilde{W}_{3} \subset \psi^{p+1}(V)$. Let $W$ be any $p$-plane containing $V$; then $\psi(W)$ intersects each of $\widetilde{W}_{1}, \widetilde{W}_{2}, \widetilde{W}_{3}$ in $(p-1)$-planes, which are not all three the same by the assumption of $\widetilde{W}_{1} \cap \widetilde{W}_{2} \cap \widetilde{W}_{3} \neq \psi_{p-1}(V)$. Thus $\psi(W) \subset \psi^{p+1}(V)$, so (ii) holds.

Corollary 4.2. Let $\varphi_{R}: \operatorname{Gr}(p, \mathscr{H}) \rightarrow \operatorname{Gr}(p, \mathscr{H})$ be induced by $R: \Lambda^{p} \mathscr{H} \rightarrow$ $\Lambda^{p} \mathscr{H}$ invertible and decomposable preserving. For $\operatorname{dim} \mathscr{H}>2 p$, there is a map $\varphi_{p-1}: \operatorname{Gr}(p-1, \mathscr{H}) \rightarrow \operatorname{Gr}(p-1, \mathscr{H})$ such that $\varphi_{R}\left(\sigma_{p}(V)\right) \subset \sigma_{p}\left(\varphi_{p-1}(V)\right)$, with $\varphi_{R}\left(\sigma_{p}(V)\right)=\sigma_{p}\left(\varphi_{p-1}(V)\right)$ if $\operatorname{dim} \mathscr{H}<\infty$. When $\operatorname{dim} \mathscr{H}=2 p$, there exists such a $\varphi_{p-1}$ either for $\varphi_{R}$ or for $* \circ \varphi$.

Proof. We apply Proposition 4.1 to $\varphi=\varphi_{R}$, which preserves adjacency. Conditions (i) and (ii) of Proposition 4.1 are equivalent to (i) ${ }^{\prime} R\left(\wedge^{p-1} V \wedge \mathscr{H}\right) \subset$ $\Lambda^{p-1} \varphi_{p-1}(V) \wedge \mathscr{H}$ and (ii) ${ }^{\prime} R\left(\wedge^{p-1} V \wedge \mathscr{H}\right) \subset \wedge^{p} \varphi^{p+1}(V)$. Now (ii) ${ }^{\prime}$ implies $\operatorname{dim} \mathscr{H}-(p-1) \leq p+1$, so we are done if $\operatorname{dim} \mathscr{H}>2 p$. If $\operatorname{dim} \mathscr{H}=2 p$, then equality holds in (i) ${ }^{\prime}$ and (ii) ${ }^{\prime}$, so (i) and (ii) become $\varphi\left(\sigma_{p}(V)\right)=\sigma_{p}\left(\varphi_{p-1}(V)\right)$ and $\varphi\left(\sigma_{p}(V)\right)=\Sigma^{p}\left(\varphi^{p+1}(V)\right)$. Since $\varphi$ is continuous, and the Schubert cycles $\sigma_{p}\left(\varphi_{p-1}(V)\right)$ and $\Sigma^{p}\left(\varphi^{p+1}(V)\right)$ represent distinct homology classes, only one of these alternatives holds for all $V \in \operatorname{Gr}(p, \mathscr{H})$. If necessary, we can compose $R$ with the endomorphism of $\bigwedge^{p} \mathscr{H}$ which induces the dual map $*$ to achieve $\varphi\left(\sigma_{p}(V)\right)=\sigma_{p}\left(\varphi_{p-1}(V)\right)$ for all $V$.

\section{ProOF OF THEOREM 1.1}

Assume $\operatorname{dim} \mathscr{V}=n \geq 2 p$, so that $\varphi_{R}\left(\sigma_{p}(W)\right)=\sigma_{p}\left(\varphi_{p-1}(W)\right)$ for all $(p-1)$-planes $W$ (if necessary, compose $\varphi_{R}$ with $*$ when $n=2 p$ ). Thus $\varphi_{p-1}$ is one-to-one. To show it is an automorphism, it suffices to show it is holomorphic, since $\operatorname{Gr}(p-1, \mathscr{V})$ is compact and connected. Coordinatize $\operatorname{Gr}(p-1, \mathscr{V})$ by letting $\psi(Z)$ be the span of the $v_{i}+\Sigma z_{i j} w_{j}$, where $v_{1}, \ldots, v_{p-1}, w_{1}, \ldots, w_{n-p+1}$ is a basis for $\mathscr{V}$. Let $W_{i}(Z)$ denote the $p$-plane spanned by $\psi(Z)$ and $w_{i}$. Then $W_{1}(Z) \cap W_{2}(Z)=\psi(Z)$ implies 
$\varphi_{p-1}(\psi(Z))=\varphi_{R}\left(W_{1}(Z)\right) \cap \varphi_{R}\left(W_{2}(Z)\right)$ is holomorphic, since the intersection has constant dimension.

Theorem 1.1 is true for $p=1$, so by induction assume $\varphi_{p-1}$ is induced by an endomorphism $S$ of $\mathscr{V}$. If $W$ is a $p$-plane in $\mathscr{V}$, then $W=\bigcap_{V \subset W} \sigma_{p}(V)$, where $\operatorname{dim} V=p-1$. Thus

$$
\begin{aligned}
\varphi(W) & =\bigcap_{V \subset W} \varphi\left(\sigma_{p}(V)\right)=\bigcap_{V \subset W} \sigma_{p-1}\left(\varphi_{p-1}(V)\right) \\
& =\bigcap_{V \subset W} \sigma_{p-1}(S V)=\bigcap_{V \subset S W} \sigma_{p-1}(V)=S W .
\end{aligned}
$$

6. $\operatorname{dim} \mathscr{H}=\infty$

When $\mathscr{H}$ is infinite dimensional, we need $R$ onto to establish that $\varphi_{p-1}$ is one-to-one, as the following example shows: Let $V$ be a $(p-1)$-plane in $\mathscr{H}$ and $\lambda$ a $(p-1)$-vector corresponding to $V$. Let $T: \wedge^{p} \mathscr{H} \rightarrow V^{\perp}$ be an injective linear operator, and define an injective linear operator $R: \Lambda^{p} \mathscr{H} \rightarrow \Lambda^{p} \mathscr{H}$ by $R \alpha=\lambda \wedge T \alpha$. Trivially $R$ preserves decomposables, yet the induced map $\varphi_{p-1}$ is constant.

Lemma 6.1. Let $\psi: \operatorname{Gr}(r, \mathscr{H}) \rightarrow \operatorname{Gr}(r, \mathscr{H})$ preserve adjacency, $r \geq 2$. Fix $Z$ an $(r-2)$-plane and $L$ an l-plane, $l \geq r-1$. If $\operatorname{dim} L \cap \psi(W) \geq r-1$ for all $W \in \sigma_{r}(Z)$, then $L \cap \psi(W) \neq 0$ for every $r$-plane $W$.

Proof. We show for any $r$-plane $W: \operatorname{dim} W \cap Z=k$ implies $\operatorname{dim} L \cap \psi(W) \geq$ $k+1$. This is trivial if $r=2$. For $r>2$, assume true for $k, 0<k \leq r-2$. Let $\widetilde{W}$ be an $r$-plane such that $\operatorname{dim} \widetilde{W} \cap Z=k-1$. There is an $r$-plane $W$ such that $\operatorname{dim} W \cap Z=k$ and $\operatorname{dim} W \cap \widetilde{W}=r-1$. Thus $\operatorname{dim} \psi(W) \cap \psi(\widetilde{W})=r-1$ and $\operatorname{dim} L \cap \psi(\widetilde{W}) \geq k=(k-1)+1$.

The main step in proving Theorem 1.2 is the following proposition:

Proposition 6.2. For $1 \leq q<p$, assume that for all $r$ with $q \leq r \leq p$ we have defined maps $\varphi_{r}: \operatorname{Gr}(r, \mathscr{H}) \rightarrow \operatorname{Gr}(r, \mathscr{H})$, where $\varphi_{p}=\varphi_{R}$, which satisfy the following for $r>q$ : (1) $\varphi_{r}$ preserves adjacency, and (2) $\varphi_{r} \sigma_{r}(V) \subset \sigma_{r}\left(\varphi_{r-1}(V)\right)$, for each $(r-1)$-plane $V$. Then $\varphi_{q}$ preserves adjacency. Furthermore, if $q>1$, then we can define $\varphi_{q-1}: \operatorname{Gr}(q-1, \mathscr{H}) \rightarrow \operatorname{Gr}(q-1, \mathscr{H})$ so that (2) holds for $r=q$.

Before proving Proposition 6.2, we use surjectivity of $R$ to show:

Lemma 6.3. Under the hypotheses of Proposition 6.2, if $Z$ is a $(q-1)$-plane and $L$ an l-plane, $l \geq q \geq 1$, then $\operatorname{dim} L \cap \varphi_{q+1}(W)<q$ for some $W \in \sigma_{q+1}(Z)$.

Proof. If not, then Lemma 6.1 shows that $L \cap \varphi_{q+1}(W) \neq 0$ for all $(q+1)$ planes $W$. If $U$ is a $(q+2)$-plane, then $\varphi_{q+2}(U)$ contains $\varphi_{q+1}(W)$ for any $(q+1)$-plane $W \subset U$, by hypothesis (2) of the Proposition, and hence $L \cap$ $\varphi_{q+2}(U) \neq 0$. Inductively we obtain that $L \cap \varphi_{R}(V) \neq 0$ for all $V \in \operatorname{Gr}(p, \mathscr{H})$. 
That is, if we let $\beta$ be an $r$-vector which represents $L$, then $R(\alpha) \wedge \beta=0$ for all $\alpha$ decomposable, and hence for all $\alpha$ in $\Lambda^{p} \mathscr{H}$. This cannot happen since $R$ is onto (and $\operatorname{dim} \mathscr{H} \geq r+p$ ).

Proof of Proposition 6.2. If $\varphi_{q}$ does not preserve adjacency, let $V_{1}$ and $V_{2}$ be adjacent $q$-planes such that $\operatorname{dim} \varphi_{q}\left(V_{1}\right) \cap \varphi_{q}\left(V_{2}\right) \neq q-1$. Since $W=V_{1}+V_{2}$ is a $(q+1)$-plane containing both $V_{1}$ and $V_{2}$, then (2) implies $\varphi_{q}\left(V_{1}\right) \subset \varphi_{q+1}(W)$, so $\varphi_{q}\left(V_{1}\right)=\varphi_{q}\left(V_{2}\right)=V_{0}$, say. Let $\widetilde{W}$ be any $(q+1)$-plane which contains $V_{1} \cap V_{2}, \widetilde{W} \neq W$, and $V$ be a $q$-plane in $\widetilde{W}$ which contains $V_{1} \cap V_{2}$ and is not contained in $W$. Let $W_{i}=V_{i}+V$ for $i=1,2$. Then $W_{1}$ and $W_{2}$ are distinct $(q+1)$-planes containing $V$, and $V_{i} \subset W_{i}$ implies that $\varphi_{q}\left(V_{i}\right) \subset \varphi_{q+1}\left(W_{i}\right)$ for $i=1,2$. Thus $\varphi_{q}(V)=\varphi_{q+1}\left(W_{1}\right) \cap \varphi_{q+1}\left(W_{2}\right)=V_{0}$ and hence $V_{0} \subset \varphi_{q+1}(\widetilde{W})$, which contradicts Lemma 6.3 (with $L=V_{0}$ ). Thus $\varphi_{q}$ preserves adjacency.

To show that (2) holds for $q>1$ : since $\varphi_{q}$ preserves adjacency, then either $\varphi_{q}\left(\sigma_{q-1}(Z)\right) \subset \sigma_{q}\left(\varphi_{q-1}(Z)\right)$ or $\varphi_{q}\left(\sigma_{q-1}(Z)\right) \subset \Sigma^{q}\left(\varphi_{q+1}(W)\right)$. If the latter, then $\varphi_{q}(V) \subset \varphi_{q+1}(W)$ for all $q$-planes $V$ which contain $Z$. But if $\widetilde{W}$ is any $(q+1)$-plane containing $Z$, let $V$ be a $q$-plane with $Z \subset V \subset \widetilde{W}$. Then $\varphi_{q}(V) \subset \varphi_{q+1}(\widetilde{W})$, so $\operatorname{dim} \varphi_{q+1}(W) \cap \varphi_{q+1}(\widetilde{W}) \geq q$ for all $\widetilde{W}$ containing $Z$, contradicting Lemma 6.3 (with $L=\varphi_{q+1}(W)$ ).

Proposition 6.4. Let $v_{1}, \ldots, v_{q}$ be independent. Then

$$
\varphi_{q}\left(\left[v_{1}, \ldots, v_{q}\right]\right)=\varphi_{1}\left(\left[v_{1}\right]\right)+\cdots+\varphi_{1}\left(\left[v_{q}\right]\right) \text {, }
$$

where $\left[v_{1}, \ldots, v_{r}\right]$ is the r-plane spanned by $v_{1}, \ldots, v_{r}$.

Proof. Since $\varphi_{1}$ preserves adjacency, then $\varphi_{1}\left(\left[v_{1}\right]\right)$ and $\varphi_{1}\left(\left[v_{2}\right]\right)$ are distinct lines in $\varphi_{2}\left(\left[v_{1}, v_{2}\right]\right)$, so the Proposition is true when $q=2$. Assume true for $q$ and $q-1$ with $2 \leq q<p$. If not true for $q+1$, then we may assume that $\varphi_{1}\left(\left[v_{q+1}\right]\right) \subset \varphi_{q}\left(\left[v_{1}, \ldots, v_{q}\right]\right)$. But $\varphi_{1}\left(\left[v_{q+1}\right]\right) \subset \varphi_{q}\left(\left[v_{1}, \ldots, v_{q-1}, v_{q+1}\right]\right)$. Thus $\varphi_{1}\left(\left[v_{q+1}\right]\right) \subset \varphi_{q-1}\left(\left[v_{1}, \ldots, v_{q-1}\right]\right)$ by definition of $\varphi_{q-1}$, so that

$$
\varphi_{q}\left(\left[v_{1}, \ldots, v_{q-1}, v_{q+1}\right]\right) \neq \varphi_{1}\left(\left[v_{1}\right]\right)+\cdots+\varphi_{1}\left(\left[v_{q-1}\right]\right)+\varphi\left(\left[v_{q+1}\right]\right),
$$

contrary to assumption.

Remark 6.5. If $V$ and $W$ are vector spaces, $T$ and $\widetilde{T}: V \rightarrow W$ linear transformations such that for each $x \in V, \widetilde{T} x=c_{x} T x$, where $c_{x} \neq 0$ is a scalar, then $\tilde{T}=c T$. (Assume $\operatorname{ker} T=0$, then use $c_{x} T x+c_{y} T y=c_{x+y}(T x+T y$ ) to get $\left.c_{x}=c_{y}.\right)$

Proof of Theorem 1.2. Fix $e_{1}, \ldots, e_{p-1}$ orthonormal in $\mathscr{H}$, and choose $\tilde{e}_{i} \in$ $\varphi_{1}\left(\left[e_{i}\right]\right)$. Let $\alpha_{i}=e_{1} \wedge \cdots \wedge e_{i}, \tilde{\alpha}_{i}=\tilde{e}_{1} \wedge \cdots \wedge \tilde{e}_{i}$, and $V_{i}$ be spanned by $e_{1}, \ldots, e_{i}$. By Proposition 6.4 , for $x \neq 0$ in $V_{p-1}^{\perp}$ there is a unique $S x \in \varphi_{1}([x])$ such that $R\left(\alpha_{p-1} \wedge x\right)=\tilde{\alpha}_{p-1} \wedge S x$. Define $S 0=0$. 
We show $S$ is linear: if $x$ and $y$ in $V_{p-1}^{\perp}$ are independent, then so are $R\left(\alpha_{p-1} \wedge x\right)=\tilde{\alpha}_{p-1} \wedge S x$ and $R\left(\alpha_{p-1} \wedge y\right)=\tilde{\alpha}_{p-1} \wedge S y$. Now $S(x+y)=$ $a S x+b S y$ since $\varphi_{1}([x+y]) \subset \varphi_{2}([x, y])$. Linearity of $R$ implies that $\tilde{\alpha}_{p-1} \wedge$ $(S(x+y)-S x-S y)=0$, so $a=b=1$ and $S(x+y)=S x+S y$. Similarly $S(c x)=c S x$.

Assume that we have defined $S$ linear on $V_{i}^{\perp}$ so that $S x \in \varphi_{1}([x])$ for $x \neq 0$ and $R\left(\alpha_{i} \wedge x_{i+1} \wedge \cdots \wedge x_{p}\right)=\tilde{\alpha}_{i} \wedge S x_{i+1} \wedge \cdots \wedge S x_{p}$; then Proposition 6.4 implies $R\left(\alpha_{i-1} \wedge x_{i} \wedge \cdots \wedge x_{p}\right)=\lambda\left(x_{i}, \ldots, x_{p}\right) \tilde{\alpha}_{i-1} \wedge S x_{i} \wedge \cdots \wedge S x_{p}$, where $\lambda\left(x_{i}, \ldots, x_{p}\right)$ is a nonzero scalar depending on $x_{i}, \ldots, x_{p}$ independent in $V_{i}^{\perp}$. From Remark 6.5, applied to fixed $x_{i}, \ldots, x_{p-1}$ with $x_{p}$ varying, we see that $\lambda$ only depends on $x_{i}, \ldots, x_{p-1}$-hence $\lambda$ is constant by symmetry.

Replacing $S$ by $1 / a S$, where $a^{p-i+1}=\lambda$, we then have

$$
R\left(\alpha_{i-1} \wedge x_{i} \wedge \cdots \wedge x_{p}\right)=\tilde{\alpha}_{i-1} \wedge S x_{i} \wedge \cdots \wedge S x_{p}
$$

for $x_{i}, \ldots, x_{p}$ independent in $V_{i}^{\perp}$. Now extend $S$ to a linear map on $V_{i-1}^{\perp}$ by setting $S e_{i}=a^{p-i} \tilde{e}_{i}$. Then $R\left(\alpha_{i-1} \wedge x_{i} \wedge \cdots \wedge x_{p}\right)=\tilde{\alpha}_{i-1} \wedge S x_{i} \wedge \cdots \wedge S x_{p}$, for $x_{i}, \ldots, x_{p}$ independent in $V_{i-1}^{\perp}$. We construct $S$ inductively so that $R\left(x_{1} \wedge\right.$ $\left.\cdots \wedge x_{p}\right)=S x_{1} \wedge \cdots \wedge S x_{p}$ for all $x_{1}, \ldots, x_{p}$ independent, and hence for all $x_{1}, \ldots, x_{p}$. Thus $R=\Lambda^{p} S$. Since $R$ is invertible, $S$ is one-to-one.

We need only show that $S$ is bounded and onto when $\mathscr{H}$ is infinite dimensional. We use that if $W_{1}$ and $W_{2}$ are disjoint finite dimensional subspaces of $\mathscr{H}$, and $P_{i}$ is projection on $W_{i}^{\perp}$, then $\Pi=P_{1}+P_{2}$ is invertible. To see this, let $W=W_{1}+W_{2}$, so that $\left.\Pi\right|_{W^{\perp}}=2 \mathrm{Id}_{W^{\perp}}$. If $x \in W$, then $P_{i} x=x-w_{i}$ where $w_{i} \in W_{i}$, hence $\Pi W \subset W$. Since $P_{i}$ is nonnegative, $\operatorname{ker} \Pi=\operatorname{ker} P_{1} \cap \operatorname{ker} P_{2}=0$, so that $\left.\Pi\right|_{W}$ is invertible.

Now fix $x_{1}, \ldots, x_{p-1}$ independent in $\mathscr{H}$. Let $P$ denote projection on the orthogonal complement of the $x_{i}$, and $Q$ projection on the orthogonal complement of the $S x_{i}$. Let $\alpha=x_{1} \wedge \cdots \wedge x_{p-1}$ and $\beta=S x_{1} \wedge \cdots \wedge S x_{p-1}$. Then $\|\beta\|\|Q S y\|=\left\|S x_{1} \wedge \cdots \wedge S x_{p-1} \wedge S y\right\| \leq\|R\|\|\alpha \wedge y\|=\|R\|\|\alpha\|\|P y\| \leq$ $\|R\|\|\alpha\|\|y\|$. If we then take $\tilde{x}_{1}, \ldots, \tilde{x}_{p-1}$ such that $x_{1}, \ldots, x_{p-1}, \tilde{x}_{1}, \ldots$, $\tilde{x}_{p-1}$ are independent, and let $\widetilde{P}$ and $\widetilde{Q}$ be defined for the $\tilde{x}_{i}$, then

$$
\|(Q+\tilde{Q}) S y\| \leq\|R\|(\|\alpha\| /\|\beta\|+\|\tilde{\alpha}\| /\|\tilde{\beta}\|)\|y\| .
$$

The boundedness of $S$ follows from invertibility of $Q+\widetilde{Q}$.

To see that $S$ is invertible: let $\mathscr{H}_{0}$ be the closure of range $S$. The range of $R$ is contained in $\Lambda^{p} \mathscr{H}_{0}$, so $\mathscr{H}_{0}=\mathscr{H}$, and $S$ has dense range. But if $S y_{n}$ converges, then $R\left(\alpha \wedge y_{n}\right)=\beta \wedge S y_{n}$ converges. This implies $\alpha \wedge y_{n}$ converges, and hence so does $P y_{n}$; similarly, so does $\widetilde{P} y_{n}$. Thus $(P+\widetilde{P}) y_{n}$ converges, which implies $y_{n}$ converges and range $S$ is closed. 


\section{ACKNOWLEDGMENT}

Before beginning, I would like to thank Ron Donagi, James Faran, Mark Green, Richard Hain, and Harald Upmeier for many useful discussions.

\section{REFERENCES}

[C] W.-L. Chow, On the geometry of algebraic homogeneous spaces, Ann. of Math. 50 (1949), 32-67.

[H] R. Hartshorne, Algebraic geometry, Springer-Verlag, New York, 1977.

[K] W. Kaup, Über die Automorphismen Graßmannscher Mannigfaltigkeiten unendlicher Dimension, Math. Z. 144 (1975), 75-96.

[T] H. Tango, On the automorphisms of Flag manifolds, Bull. Kyoto Univ. of Ed., B, 49 (1976), $1-9$.

Department of Mathematics, State University of New York at Buffalo, Buffalo, NEW YORK 14214 